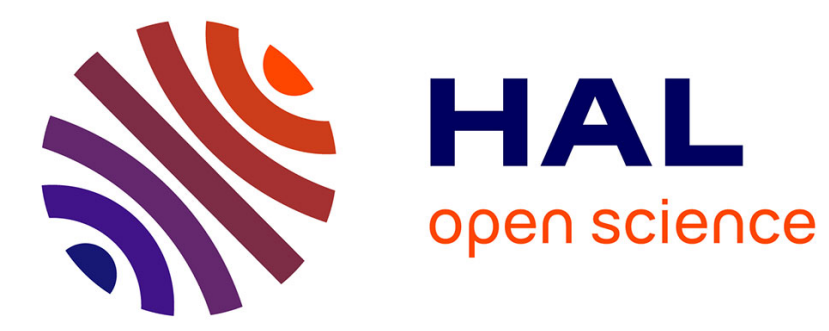

\title{
Distribution of zeros in the rough geometry of fluctuating interfaces
}

\author{
Arturo L Zamorategui, Vivien Lecomte, Alejandro B. Kolton
}

\section{To cite this version:}

Arturo L Zamorategui, Vivien Lecomte, Alejandro B. Kolton. Distribution of zeros in the rough geometry of fluctuating interfaces. 2015. hal-01262599

\section{HAL Id: hal-01262599 \\ https://hal.science/hal-01262599}

Preprint submitted on 26 Jan 2016

HAL is a multi-disciplinary open access archive for the deposit and dissemination of scientific research documents, whether they are published or not. The documents may come from teaching and research institutions in France or abroad, or from public or private research centers.
L'archive ouverte pluridisciplinaire HAL, est destinée au dépôt et à la diffusion de documents scientifiques de niveau recherche, publiés ou non, émanant des établissements d'enseignement et de recherche français ou étrangers, des laboratoires publics ou privés. 


\title{
Distribution of zeros in the rough geometry of fluctuating interfaces
}

\author{
Arturo L. Zamorategui and Vivien Lecomte \\ Laboratoire Probabilités et Modèles Aléatoires (UMR CNRS 7599), \\ Université Pierre et Marie Curie and Université Paris Diderot, 75013 Paris, France
}

\author{
Alejandro B. Kolton \\ CONICET-Centro Atómico Bariloche and Instituto Balseiro (UNCu), 8400 S.C. de Bariloche, Argentina
}

(Dated: December 14, 2015)

\begin{abstract}
We study numerically the correlations and the distribution of intervals between successive zeros in the fluctuating geometry of stochastic interfaces, described by the Edwards-Wilkinson equation. For equilibrium states we find that the distribution of interval lengths satisfies a truncated Sparre-Andersen theorem. We show that boundary-dependent finite-size effects induce non-trivial correlations, implying that the independent interval property is not exactly satisfied in finite systems. For out-of-equilibrium non-stationary states we derive the scaling law describing the temporal evolution of the density of zeros starting from an uncorrelated initial condition. As a by-product we derive a general criterion of the Von Neumann's type to understand how discretization affects the stability of the numerical integration of stochastic interfaces. We consider both diffusive and spatially fractional dynamics. Our results provide an alternative experimental method for extracting universal information of fluctuating interfaces such as domain walls in thin ferromagnets or ferroelectrics, based exclusively on the detection of crossing points.
\end{abstract}

\section{INTRODUCTION}

Persistence and its related first-passage properties have been of great interest in recent years both in mathematics and in physics, going from simple models such as the $d$-dimensional random walk in its discrete and continuous versions ${ }^{1,2}$ and the non-Markovian acceleration process $^{3-5}$, or the $d$-dimensional Ising and Potts model at zero temperature with Glauber dynamics ${ }^{6,7}$ to systems with many degrees of freedom such as the diffusion equation of fields ${ }^{8}$, solid-on-solid surface growth models ${ }^{9}$ and fluctuating interfaces. ${ }^{10-12}$ (For a recent review on persistence in non-equilibrium systems see Ref. [13].)

The notion of spatial persistence (resp. temporal persistence) in these systems refers to a property that does not change up to a distance $x$ (resp. up to a time $t$ ), being of special interest the probability distributions $P(\ell)$ and $P(\tau)$ of the corresponding distances or time intervals between the successive changes. Due to its possible applications, both spatial persistence ${ }^{14,15}$ and temporal persistence $^{16}$ of rough fluctuating interfaces have been investigated. In this case, the property whose change is monitored is the sign of the interface height with respect to some reference line, and thus $\ell$ refers to the distances between successive zeros of an instantaneous configuration, and $\tau$ to the duration between successive zeros of the height at a fixed point in space. In a slightly different context ${ }^{17,18}$, the persistent property refers to the fraction of (for instance Ising) spins that have never flipped up to time $t$. In order to reach a general understanding of these kind of phenomena it is useful to analyze model systems, such as Markovian ${ }^{14,18,19}$ or non-Markovian ${ }^{16,20}$ Gaussian process with zero mean and unit variance $Y(x)$ (resp. $Y(t))$. In these cases the strategy to get $P(\ell)$ (resp. $P(\tau)$ ) is to extract it from the two-point correlation function $C\left(x, x^{\prime}\right)=\left\langle Y(x) Y\left(x^{\prime}\right)\right\rangle$ (resp. $\left.C\left(t, t^{\prime}\right)=\left\langle Y(t) Y\left(t^{\prime}\right)\right\rangle\right)$ by means of the probability that the process $Y(x)$ (resp. $Y(t))$ has the same sign for two different points in space $x$ and $x^{\prime}$ (resp. for two different times $t$ and $t^{\prime}$ ) $14,16,18,19$, via approximation methods such as the independent interval approximation (IIA). ${ }^{21,22}$ Even for some simple processes, the derivation of $C\left(x, x^{\prime}\right)$ (resp. $C\left(t, t^{\prime}\right)$ ) is not a trivial task. In the case of the Brownian motion and of the acceleration process, a suitable transformation maps these two processes into stationary Gaussian processes for which the two-time correlation function takes a simpler form - it depends only on the difference between the two times considered. ${ }^{23}$ Some generalizations have been investigated such as the probability of having $N$ zeros between two times $t$ and $t^{\prime}$ for Markovian ${ }^{19}$ and non-Markovian processes. ${ }^{16,24,25}$

The persistence probability in different contexts in the large time or large distance limit is found to follow a power law $Q(\ell) \sim \ell^{-\theta}$ where $\theta$ is the persistence exponent (here again the interval $\ell$ might refer to time or spatial intervals). Even for simple diffusion it was shown that the persistence probability has a nontrivial persistence exponent $\theta \cdot{ }^{19}$ Concerning fluctuating interfaces and surface growth, their persistence and their associated first-passage properties have been of considerable interest in the physical literature. ${ }^{10}$ Starting from a flat interface and letting it evolve according to a linear Langevin fractional differential equation $\partial_{t} u(x, t)=$ $-c\left(-\nabla^{2}\right)^{\alpha} u(x, t)+\eta(x, t)$ where $\alpha$ relates to the roughness exponent $\zeta$ as $\alpha=\frac{1}{2}+\zeta$ (which can be associated with non-local harmonic elastic forces on $u(x, t)$ in general), it is found that the behavior of the temporal persistence probability $Q\left(t_{0}, t\right)$, understood as the probability that the interface stays above (or below) its initial value at $t_{0}$ on the interval $\left[t_{0}, t_{0}+t\right]$, depends strongly on the initial conditions. Two limiting cases were considered for the temporal persistence in Ref. [26]: for $t_{0}=0$, the 
so-called transient or coarsening persistence probability $Q_{0}(t)=Q\left(t_{0}=0, t\right)$ is found to decay as $Q_{0}(t) \sim t^{-\theta_{0}}$ for $t \rightarrow \infty$ with $\theta_{0}$ a non trivial exponent. On the other hand, for $t_{0} \rightarrow \infty$ the steady-state persistence probability behaves as $Q_{s}(t)=\lim _{t_{0} \rightarrow \infty} q\left(t_{0}, t\right) \sim t^{-\theta_{s}}$ with $\theta_{s} \neq \theta_{0}$. Extensive numerical simulations for the calculation of temporal persistence in surface growth processes belonging to different universality classes have been performed in Ref. [20]. The authors calculate separately the positive and negative persistent exponents, i.e. the exponents associated to the intervals where the surface remains above or below certain level, respectively, both transient $\theta_{0}^{ \pm}$and steady-state exponents $\theta_{s}^{ \pm}$. Moreover, the authors show that $\theta_{s}^{+}$and $\theta_{s}^{-}$are always different for surfaces simulated from nonlinear equations with broken mirror symmetry, since in this case the surface tends to spend more time on the positive or negative values. Results have been obtained regarding spatial persistence of surface growth processes as well. ${ }^{15}$

In this paper we investigate finite-time and finite-size effects for different observables of the stochastic dynamics of $\zeta=1 / 2$ Edwards-Wilkinson interfaces with periodic boundary conditions, which can affect the statistics of its crossing zeros (we leave the study of the general nonMarkovian case for a forthcoming paper ${ }^{27}$ ). Since accessible systems are finite, both experimentally and numerically, this kind of study is of importance for the numerical validation of analytical results and their approximations ${ }^{28}$. We show in particular that finite-size effects and boundary conditions can affect the shape of the steadystate spatial distribution of intervals $P(\ell)$, and the validity of the IIA for large $\ell$. Since the Edwards-Wilkinson interface is linear and statistically invariant by a change of sign $u(x, t) \mapsto-u(x, t)$, we expect that the steadystate persistence exponents for the positive and the negative intervals are equal, i.e. $\theta_{s}^{+}=\theta_{s}^{-}=\theta$. Further, we relate the distribution of intervals to the first-passage distribution of a random walk. This mapping between Gaussian interfaces with height $u(x, t)$ at point $x$ and time $t$ and the stochastic process evolving via $d^{n} X / d t^{n}=\eta(t)$ was studied in Ref. [14] with the correspondence $u \mapsto X$ and $x \mapsto t$. A link between the discretized stationary interface and a discrete random walk is thus made by means of the Sparre-Andersen theorem. ${ }^{29}$ This theorem describes the persistence probability $P_{0}(n)$ of a random walker to stay positive (or negative) up to a step $n$ starting in 0 . We discuss as well the influence of the boundary conditions on the correlator of consecutive jumps in the interface.

We also analyze the statistics of crossing points in nonstationary states, starting from an uncorrelated configuration. While steady states can be directly sampled with their equilibrium Boltzmann weight, non-stationary states are obtained by numerically solving the dynamics. To this end we first derive a numerically stable scheme by generalizing the Von Neumann stability criterion ${ }^{30}$ for deterministic differential equations to the general case of the Langevin spatially fractional differential equation describing interfaces with local and non-local elasticities. Although we present numerical results for interfaces with roughness exponent $\zeta=1 / 2$, the stability condition we derive is general and relates the time step used for the simulations with the roughness exponent $\zeta$ and a parameter related to the time discretization scheme. In this context, Itō and Stratonovich discretizations are just two special cases. ${ }^{31,32}$ By solving the non-stationary dynamics within this scheme we obtain the scaling law describing the temporal evolution of the density of zeros towards the steady-state results previously analyzed.

The paper is organized as follows. In Section II we present our model and the main observables considered. In Section III we focus on the stationary state of discrete interfaces with periodic boundary conditions, analyzing in detail the finite-size effects in the distribution and spatial correlations of intervals between consecutive zeros. In Section IV we focus on non-stationary interfaces starting from a flat interface. We first compute exactly the structure factor as a function of time and we obtain that it keeps track of the choice of convention for the time discretization. From this expression we extract a general Von-Neumann like stability criterion for the stochastic dynamics of interfaces. The numerical results for the evolution of the structure factor of the interface and for the density of zeros yield time-dependent scaling laws describing the approach to the steady state results. Finally, in Section V we give our conclusions and perspectives.

\section{MODEL AND OBSERVABLES}

In this section we introduce the model of the interface we are going to study. Although we focus in this paper on the discrete version of the system which is the one numerically accessible, we present first the continuous solution of the Langevin equation introduced above, for completeness. We also present the observables of interest, namely the length of the intervals between successive zeros, the correlation function for the intervals, the structure factor and the density of zeros.

To start with, we consider a fluctuating interface of size $L$ with height $u(x, t)$ at position $x$ and time $t$ measured with respect to the origin. The function $u(x, t)$ satisfies the linear Langevin equation

$$
\partial_{t} u(x, t)=-c\left(-\nabla^{2}\right)^{\alpha} u(x, t)+\eta(x, t)
$$

where the exponent $\alpha$ of the Laplacian is related to the roughness exponent $\zeta$ as $\alpha=\frac{1}{2}+\zeta$ and the thermal noise $\eta(x, t)$ is defined with mean $\langle\eta(x, t)\rangle=0$ and variance $\left\langle\eta(x, t) \eta\left(x^{\prime}, t^{\prime}\right)\right\rangle=2 T \delta\left(x-x^{\prime}\right) \delta\left(t-t^{\prime}\right)$. We consider periodic boundary conditions such that $u(0, t)=u(L, t)$. We will be interested in the fluctuating dynamics of the interface starting from the flat initial condition $u(x, t=0)=$ 0 . The general solution of Eq. (1) can thus be written in Fourier space as

$$
u(q, t)=\int_{0}^{t} e^{-c q^{\alpha}\left(t-t^{\prime}\right)} \eta_{q}\left(t^{\prime}\right) d t^{\prime}
$$


where the Fourier transform is defined as $u(q, t)=$ $\int_{0}^{L} e^{-i q x} u(x, t) d x$, and thus $u(q, t=0)=0$. The Fourier noise has mean value $\left\langle\eta_{q}(t)\right\rangle=0$ and variance $\left\langle\eta_{q}(t) \eta_{q^{\prime}}\left(t^{\prime}\right)\right\rangle=2 T L \delta\left(t-t^{\prime}\right) \delta_{q q^{\prime}}$. We will be interested both in the non-stationary and the steady-state solutions of Eq. (1) which is reached at long times $t \sim L^{2}$.

At a given time $t$, the average height is $\bar{u}(t)=$ $\frac{1}{L} \int_{0}^{L} u(x, t) d x$ where $L$ is the size of the interface. We define a zero as the crossing point of the interface with its mean value $\bar{u}(t)$, i.e. the points such that $u(x, t)-\bar{u}(t)=$ 0 . Although the Fourier modes are independent, as shown by (2), the zeros are defined in real space and thus have a non-trivial statistics. For simplicity we can fix the mean value of the interface to zero, which is equivalent to fixing the amplitude of the first mode to zero $u(q=0, t)=0$. Under this assumption a zero of $u(x, t)$ is identified with a change of sign.

In the following we work on a lattice of $L$ sites with spacing $\Delta x=1$ and we denote the height of the interface as $u_{x}(t)$ with $x=1,2, \ldots, L$ (See Fig. 1 ). We focus on the Fourier transform of the height defined as $u_{q_{k}}(t)=\sum_{x=0}^{L-1} u_{x}(t) e^{-i q_{k} x}$, with $q_{k}=2 \pi k / L$ and $k=0, \ldots, L / 2+1$, as an abuse of notation we will omit the subscript $k$ in the following. $u_{q}(t)$ satisfies the general discretized equation (12) introduced below. Periodic boundary conditions and $u_{q=0}(t)=\sum_{x=0}^{L-1} u_{x}(t)=0$ are assumed. The identification of the zeros of the interface on a lattice is not trivial as in the continuous case. In Section III A we discuss in detail such way of detecting the zeros which in turn is fundamental for a proper description of the intervals. After detecting a change of sign of the interface from one site to the next, an appropriate method must be defined so that there is not ambiguity in choosing what site contains the zero (see Fig. 1). In this paper we define a zero to be the site immediately to the left of the crossing at which the height changes sign. The intervals between consecutive zeros are denoted by $\ell_{i}$ with $i=1, \ldots, N$ and by $N$ the number of intervals.

In the stationary state of the interface, we look at the distribution of the lengths of the spatial intervals $P(\ell)$, which will be defined carefully in the next section. In the following, when talking about an interval of the interface we refer to the length $\ell$ of a spatial interval defined on the lattice with spacing $\Delta x=1$. The distribution of the intervals $\ell$ is obtained by direct sampling of stationary configurations. We will also study the spatial correlations of the intervals where the correlation function is defined as $C(r)=\left\langle\ell_{i} \ell_{i+r}\right\rangle-\left\langle\ell_{i}\right\rangle\left\langle\ell_{i+r}\right\rangle$ for two intervals $\ell_{i}$ and $\ell_{i+r}$ averaged over all the (ordered) pairs of intervals. Additionally, we discuss how periodic boundary conditions induce correlations in the jumps even for a random walk with increments $\eta_{x}$ with mean $\left\langle\eta_{x}\right\rangle=0$ and variance $\left\langle\eta_{x} \eta_{x}^{\prime}\right\rangle=2 T \delta_{x x^{\prime}}$. In the non-stationary state we are interested in the evolution of the density of zeros $\rho(t)=N / L$, where $N$ is the number of zeros and $L$ is the size of the lattice. Such density of zeros is extracted from the dynamics of the interface evolving from a flat interface at time $t=0$. Concerning the interface, we analyze the structure factor defined as $S_{q}(t)=\left\langle u_{q}^{*}(t) u_{q}(t)\right\rangle$ where the $u_{q}(t)$ is the Fourier transform of $u_{x}(t)$, with $q=2 \pi k / L$ and $k=0, \ldots, L / 2+1$.

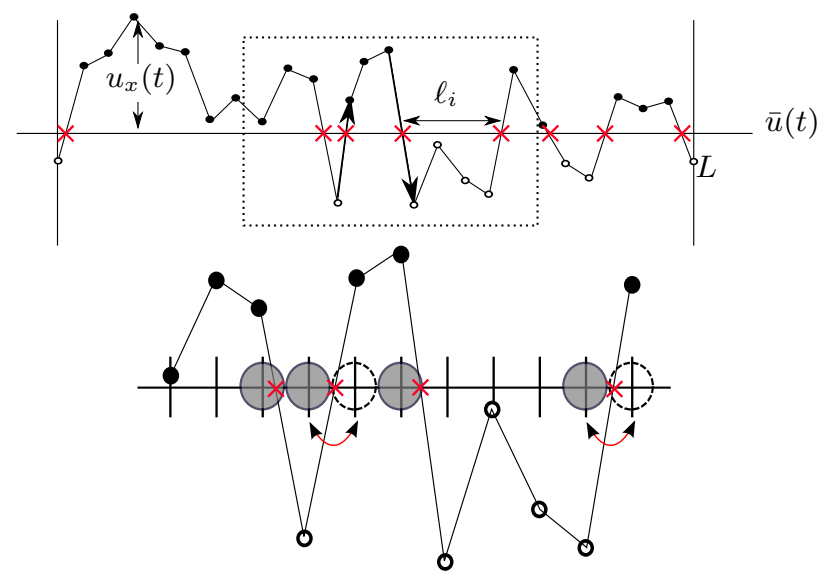

FIG. 1: An interface with periodic boundary conditions and height $u_{x}(t)$ measured with respect to its average value $\bar{u}(t)$ defined on a lattice with $L$ sites. The zeros are the points where the interface intersects its mean value $\bar{u}(t)$ given by the crosses. Such zeros divide the lattice in $N$ intervals of lengths $\ell_{i}, i=1, \ldots, N$. Since we work in discrete space, the detection of the zeros can be either selecting the site on the left of the point where the interface crosses its average value (filled circles at the bottom) or by choosing the site that is closer to the crossing point (empty circles). Notice how the choice of the method modifies the length of the intervals between consecutive zeros. In this case the second and the fourth gray zero are on the next site on the right if the nearest site was chosen. Moreover, when choosing a zero as the nearest site to the crossing point, one can find two zeros at the same site which in turn allows the existence of intervals of length $\ell=0$.

\section{STATIONARY STATE}

To start with, we analyze the stationary features of the interface. In this section we refer to the height of the interface at a certain point $x$ simply as $u_{x}=u_{x}(t \rightarrow \infty)$ by omitting the time dependence. We begin with a brief discussion about the persistence properties of a discrete random walk which will be naturally extended to the distribution of the intervals between consecutive zeros. Later on, we study numerically the correlations between such intervals and we present a scaling function for such correlations. We conclude this Section by describing how the boundary conditions are determinant for the appearance of correlations. In particular we look at the correlation of the spatial increments of the interface. 


\section{A. Distribution of intervals as first-passage distribution of a random walk.}

Let us denote $u_{n}$ the position of an unbiased random walker at step $n$. Then, the persistence probability for this random walker to stay positive up to step $n$, having started in $u_{0}>0$, is denoted by $Q\left(u_{0}, n\right)$. Similarly, the probability that the random walker reaches the origin in exactly $n$ steps starting in $u_{0}>0$ is $P\left(u_{0}, n\right)=Q\left(u_{0}, n\right)-Q\left(u_{0}, n+1\right)$, which is known as the first-passage probability. When the random walk is defined in continuous space and time $u(t)$ with $t \geq 0$, the first-passage probability $P(t)$ is defined as the probability density of the time at which the random walker changes sign, i.e. the probability that the process has a zero at time $t$. In this case $P(t)=-d Q(t) / d t$, with $Q(t)$ the persistence probability that the walker stays positive between time 0 and time $t$. Note that already for this simple process in the discrete case the definition of a zero is not trivial. We will discuss in detail how to detect the zeros when the process is discrete when we describe the relation of the discrete random walk with the fluctuating interface.

Consider now the jump distribution of the random walk given by the function $\phi(\eta)$ which we assume symmetric and continuous. The persistence probability $Q\left(u_{0}, n\right)$ is the probability that $u_{i} \geq 0$ for all $i=1, \ldots, n$ having started in $u_{0}$. By considering the first step of the walker with a stochastic jump from $u_{0}$ to $u_{1}$ and letting evolve the random walker for $n-1$ steps with the jumps $\eta_{i}=u_{i}-u_{i-1}$ being independent and identically distributed, we can write a backward equation for $Q\left(u_{0}, n\right)$ as

$$
Q\left(u_{0}, n\right)=\int_{0}^{\infty} Q\left(u_{1}, n-1\right) \phi\left(u_{1}-u_{0}\right) d u_{1}
$$

with initial condition $Q\left(u_{0}, 0\right)=1$ for all $u_{0} \geq 0$.

Although $Q\left(u_{0}, n\right)$ depends explicitly on the jump distribution $\phi(\eta)$, as seen in Eq. (3), it can be shown that under our previous assumptions $Q(0, n)$ is independent of $\phi(\eta)$ (See Ref. [16] and references therein). Moreover $Q(0, n)$ takes the following simple form

$$
Q(0, n)=\left(\begin{array}{c}
2 n \\
n
\end{array}\right) 2^{-2 n}
$$

which is the celebrated Sparre-Andersen theorem. ${ }^{29}$

In the limit of large $n$, the persistence probability $Q(0, n)$ behaves as $\sim n^{-\theta}$ with $\theta=1 / 2$ the persistence exponent. This exponent is universal in the sense that even when $Q\left(u_{0}, n\right)$ depends on $\phi, \theta$ does not depend neither on $u_{0}$ nor on $\phi$. It is easy to see that the first-passage probability $P(0, n)$ behaves as $\sim n^{-3 / 2}$ at large $n$.

The excursion made by a discrete random walker starting at $u_{0}=u_{n=0}$ that remains positive up to step $n$ and becomes negative at step $n+1$ resembles the behavior of the interface in a given interval starting from $u_{0}=u_{x=0}(t=0)$. With this image in mind, we can think of the number of steps $n$ during which the random walker does not change sign as the length $\ell$ of the intervals generated by the zeros of the interface (See Fig. 1). This suggests that the persistence probability $Q\left(u_{0}, n\right)$ defined for the random walk might describe well the probability $Q\left(u_{0}, \ell\right)$ that the interface $u_{x}$ stays above its mean height for a distance $x=\ell$. At this point, even if this idea seems reasonable, we are not ready to extend the first-passage probability $P(n)$ of the discrete random walk to the probability $P(\ell)$ that the interface has an interval of length $\ell$. First let us discuss how to define a zero when working on a lattice. Note that in this case a zero can no longer be identified just by a change of sign of $u_{x}$ (See Fig. 1). Let us imagine that $u_{x-1}(t)>0$ at $x-1, u_{x}<0$ on the next site and $u_{x+1}>0$ again on the next, then it exists $x^{\prime} \in \mathbb{R}$ in the interval $(x-1, x)$ such that $u_{x^{\prime}}(t)=0$ and $x^{\prime \prime}$ in the interval $(x, x+1)$ such that $u_{x^{\prime \prime}}=0$. Moreover let us assume that both $x^{\prime}$ and $x^{\prime \prime}$ are closer to $x$, in this case we could define a zero to be the site on the lattice that is closer to the crossing point. If this were the case, we would find two zeros on the same site $x$ in our example, therefore an interval of length $\ell=0$ is found between these two zeros. Another way of finding the zeros would be choosing always the site on the left (or on the right) to the crossing point, this would leave in this case a zero on the site $x-1$ and other zero on $x$, and thus an interval of length $\ell=1$ between these zeros. The later method, which we will adopt, produces more simple results for which the Sparre-Andersen theorem also applies in spite of the small correlations induced by periodicity.

Always choosing the site on the left (by symmetry we obtain the same results if we choose the site on the right) prevents us of having intervals of length $\ell=0$ which however are allowed in the calculation of the persistence function $Q\left(u_{0}, n\right)$ of a random walk. ${ }^{26}$ In our case, if such intervals of length $\ell=0$ were allowed, we observe, in comparison with the Sparre-Andersen theorem, that the statistics for intervals of length $\ell=1$ changes and becomes more sensitive to the correlations induced by periodic boundary condition, that are present even at short scales (see subsection III B). Another issue to take into account is that Eq. (4) is obtained by choosing the initial condition of the random walker as $u_{0}=0$. However, for our interface with continuous displacements it is rare to have an interval that starts exactly at $u_{0}=0$. The effect of the discretization for a random walk on a semi-infinite domain produces that the average return time to the origin (first-passage time) is finite. This is in contrast with the continuous random walk for which such mean interval is zero due to the infinite number of crossings that follows after the random walker changes sign for the first time before making a long excursion. Moreover, the periodic boundary conditions constrain the sum of the lengths of the intervals to be exactly $L$ the size of the lattice given, i.e. $\sum_{k}^{N} \ell_{k}=L$, with $N$ the number of intervals which is always even.

Under the previous assumptions, the probability $p(\ell)$ of having an interval of length $\ell \geq 1$ is in a good agree- 
ment with the first-passage probability of a discrete random walk with initial condition $u_{0}=0$ after normalization which gives a modified Sparre-Andersen theorem

$$
p(\ell)=\frac{1}{Z}(Q(\ell)-Q(\ell+1))
$$

Here $Q(\ell)=\left(\begin{array}{c}2 \ell \\ \ell\end{array}\right) 2^{-2 \ell}$ as in Eq. (4) and $Z$ is a normalization factor

$$
\begin{aligned}
Z=\sum_{\ell=1}^{\ell_{\max }} P(\ell) & =\sum_{\ell=1}^{\ell_{\max }}[Q(\ell)-Q(\ell+1)] \\
& =\frac{1}{2}-2^{-2 L}\left(\begin{array}{c}
2 L \\
L
\end{array}\right)
\end{aligned}
$$

which rules out intervals of length $\ell=0$ and $\ell>\ell_{\max }=$ $L-1$.

By direct-sampling of stationary configurations we can obtain numerically the distribution of the intervals for different system sizes $L$ (see Fig. 2). The stationary configurations in Fourier space for an interface with roughness exponent $\zeta$ present a Gaussian distribution and can thus be directly obtained from the structure factor $S_{q}=S_{q}(t \rightarrow \infty)=\left\langle u_{q}^{*} u_{q}\right\rangle$.

For our Edwards-Wilkinson interface of interest, we have used the large-scale expression $S_{q}=\frac{T L}{c q^{2}}$ with $T L=$ $\tilde{T}=0.1$ and $c=1$. We discuss in Section IV other choices of structure factors including e.g. finite-size corrections.

This is done by generating random Gaussian amplitudes $u_{q}$ with zero mean and variance proportional to $S_{q}$ as explained in Ref. [33]. We find that the histograms of the intervals $\ell$ for different $L$ satisfy, up to corrections due to the discretization, the modified Sparre-Andersen theorem (5) as shown in Fig. 2, at least in the region where the length of the intervals is much smaller than $L / 2$. Two comments are in order. The method of defining the zeros discussed in the previous subsection influences the results for $\ell>1$ : in fact, our convention for the definition of the location of zeros proves to be in surprising good agreement with the Sparre-Andersen theorem, with the normalization factor described in (6). One could expect that correlations induced by the periodic boundary conditions would render this result invalid, but this is not the case. However, if one takes other definitions for the locations of zeros, the correspondence would not hold. Second, for large $\ell$ but smaller than the system size, in particular for $\ell<L / 2$, the intervals satisfy a power law behavior $P(\ell) \sim \ell^{-\gamma}$ with $\gamma=3 / 2$. Since this exponent $\gamma$ for the distribution of intervals is related to the steadystate persistence exponent $\theta$ as $\gamma=\theta+1$, we obtain the expected persistence exponent $\theta=1 / 2$ for fluctuating interfaces with roughness exponent $\zeta=1 / 2$ or the persistence exponent of a discrete random walk.

For $\ell$ above $L / 2$, the effects of periodicity are very strong and this induces a cut-off at this lengthscale as observed in the numerical results. A corresponding scaling law can in fact be found for the distribution of the intervals for large values of $\ell$ which turns out to be $p(\ell) \sim L^{-3 / 2} \hat{p}(\ell / L)$, with $\hat{p}(x)$ decaying rapidly for $x \gg 1$, and $\hat{p}(x) \sim x^{-3 / 2}$ for intermediate $x \ll 1$. Fig. 2 shows that intervals of length $\ell>L / 2$ are indeed very rare, it also shows that the scaling is valid in the region for large $\ell<L$.

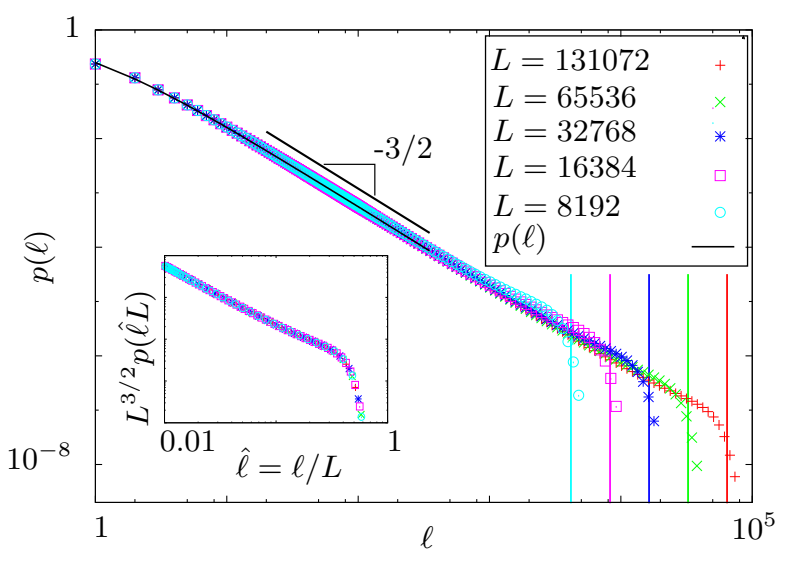

FIG. 2: Normalized histogram of the distances between consecutive zeros $\ell$ for different sizes of the system compared to the modified Sparre-Andersen theorem $p(\ell)=(q(\ell)-$ $q(\ell+1)) / Z$ with $Z$ the normalization factor given by Eq. (6) and $q(\ell)=\left(\begin{array}{c}2 \ell \\ \ell\end{array}\right) 2^{-2 \ell}$ which is in good agreement even for small values of $\ell$. Vertical lines show the values of $L / 2$ for $L=8192,16384,32768,65536,131072$ (in cyan, fuchsia, blue, green and red respectively, in the color version). Inset shows the tail of the distribution for $L$ large (which are the points for which Sparre-Andersen is no longer valid) with its points calculated from the average of the original data taken on logarithmic bins. In the limit $L \rightarrow \infty$ Sparre-Andersen is always satisfied. A scaling law is found for the probability of the intervals for large values of $\ell$ for which all the curves superpose. This law is $p(\ell, L) \sim L^{-3 / 2} \hat{p}(\ell / L)$ as explained in the text .

\section{B. Correlations of increments}

For a 1d interface drawn from the steady state described with height $u_{x}$ at a distance $x$ with initial condition $u_{0}=0$, the consecutive increments are decorrelated since the process is Brownian along the spatial direction $x$ and thus Markovian. By comparing two simple examples, we investigate first how periodic boundary conditions induce correlations on the increments even for this simple process. We start first with the analysis of the correlator of the increments of a random walk attached in one end and free in the other to find afterwards the correlator of the process itself. Then we impose boundary conditions and find the correlator of the jumps for this constrained system which turn out to present longrange correlations. We underline that the discretization in space and the periodic boundary conditions help us to understand the correlations between intervals. By the 
end of this Section we define the correlation function for the intervals in the stationary state and present some numerical results.

\section{Example: Interface attached in one end and free in the other.}

In this case the interface is attached at the origin $u_{0}=$ 0 but it is free at the other extreme. The height at every position is determined by $u_{x+1}=u_{x}+\eta_{x}$ where the noise is distributed as $\left\langle\eta_{x}\right\rangle=0$ and second moment $\left\langle\eta_{x} \eta_{x^{\prime}}\right\rangle=$ $2 T \delta_{x x^{\prime}}$, thus the jumps are uncorrelated. Therefore the process at position $x$ is defined as

$$
u_{x}=\sum_{x^{\prime}=0}^{x-1} \eta_{x^{\prime}}
$$

or

$$
\vec{u}=\mathbb{M} \vec{\eta},
$$

with $\mathbb{M}$ a lower triangular matrix.

The probability of a history is obtained from that of the noise, which is Gaussian, and for our choice of correlations above reads as follows

$$
P\left(\eta_{0}, \eta_{1}, \ldots, \eta_{L-1}\right) \propto \exp \left(-\frac{1}{2 T} \vec{\eta} \mathbb{K}_{\eta_{x}} \vec{\eta}\right)
$$

with $T$ the physical temperature of the process and $\mathbb{K}_{\eta_{x}}$ the identity matrix of dimension $L \times L$. To see how the process $\vec{u}$ is distributed we can get $\vec{\eta}$ from Eq. (7) and insert it in Eq. (8) as follows

$$
\begin{aligned}
P(\vec{u}) & \propto \exp \left(-\frac{1}{2 T} \vec{u}\left(\mathbb{M}^{-1}\right)^{\dagger} \mathbb{K}_{\eta_{x}} \mathbb{M}^{-1} \vec{u}\right) \\
& =\exp \left(-\frac{1}{2 T} \vec{u} \mathbb{K}_{u_{x}} \vec{u}\right) \\
& =\exp \left(-\frac{1}{2 T} \sum_{x=0}^{L-1}\left(u_{x+1}-u_{x}\right)^{2}\right)
\end{aligned}
$$

since the transformation (7) has unit Jacobian. The matrix $\mathbb{K}_{u_{x}}=\left(\mathbb{M}^{-1}\right)^{\dagger} \mathbb{K}_{\eta_{x}} \mathbb{M}^{-1}$ has the form of a discrete Laplacian

$$
\mathbb{K}_{u_{x}}=\left(\begin{array}{ccccc}
2 & -1 & 0 & \cdots & 0 \\
-1 & \ddots & \ddots & \ddots & \vdots \\
0 & \ddots & \ddots & \ddots & 0 \\
\vdots & \ddots & \ddots & 2 & -1 \\
0 & \cdots & 0 & -1 & 1
\end{array}\right)
$$

The Fourier transform of $\vec{u}$ can be also expressed in matrix form as $\vec{u}_{q}=\mathbb{F} \vec{u}_{x}$, where we leave the subindex to identify $\vec{u}$ from its Fourier transform. The elements of the matrix $\mathbb{F}$ are $\mathbb{F}_{j k}=\exp \left(-\frac{2 i \pi}{L} j k\right)$ and the elements of its inverse are $\left(\mathbb{F}^{-1}\right)_{j k}=\frac{1}{L} \exp \left(\frac{2 i \pi}{L} j k\right)$ Similarly, we can define the Fourier transform of the noise as $\vec{\eta}_{q}=\mathbb{F} \vec{\eta}_{x}$.

\section{Example. Interface with periodic boundary conditions}

To determine the correlations induced by the periodic boundary conditions (p.b.c), we now follow the procedure of the previous example, but backwards. We will start from the known distribution of the interface position and deduce from it the correlator of its elementary increments $\eta_{x}$. For p.b.c., the probability of a history in Fourier space $\vec{u}_{q}$ is (See Section IV for details):

$$
\begin{aligned}
P\left[\vec{u}_{q}\right] & =\exp \left(-\frac{1}{2 T} \sum_{q} 2(1-\cos q) u_{q}^{*} u_{q}\right) \\
& =\exp \left(-\frac{1}{2 T} \vec{u}_{q}^{*} \mathbb{K}_{q} \vec{u}_{q}\right)
\end{aligned}
$$

with $q=\frac{2 \pi k}{L}, \vec{u}_{q}=\mathbb{F} \vec{u}_{x}$ the Fourier transform of $\vec{u}_{x}$ and $\sum_{q}$ running over the Fourier index $k=0 \ldots L-1$.

The probability of a history $\vec{u}_{x}$ with p.b.c takes a similar form as in the previous example. We substitute $\vec{u}_{q}$ in Eq. (9) in terms of $\vec{u}_{x}$ as

$$
\begin{aligned}
P^{\mathrm{pbc}}\left[\vec{u}_{x}\right] & \propto \exp \left(-\frac{1}{2 T} \vec{u}_{x} \mathbb{F}^{\dagger} \mathbb{K}_{q} \mathbb{F} \vec{u}_{x}\right) \\
& =\exp \left(-\frac{1}{2 T} \vec{u}_{x} \mathbb{K}_{u_{x}}^{\mathrm{pbc}} \vec{u}_{x}\right)
\end{aligned}
$$

where

$$
\mathbb{K}_{u_{x}}^{\mathrm{pbc}}=\left(\begin{array}{cccccc}
2 & -1 & 0 & \cdots & 0 & -1 \\
-1 & \ddots & \ddots & \ddots & & 0 \\
0 & \ddots & \ddots & \ddots & \ddots & \vdots \\
\vdots & \ddots & \ddots & \ddots & \ddots & 0 \\
0 & & \ddots & \ddots & \ddots & -1 \\
-1 & 0 & \cdots & 0 & -1 & 2
\end{array}\right) .
$$

Then in Eq. (10) we express $\vec{u}_{x}=\mathbb{M} \vec{\eta}_{x}$ in terms of $\vec{\eta}_{x}$ as follows

$$
\begin{aligned}
P^{\mathrm{pbc}}\left[\vec{\eta}_{x}\right] & \propto \exp \left(-\frac{1}{2 T} \vec{\eta}_{x}(\mathbb{F M})^{\dagger} \mathbb{K}_{q}^{\mathrm{pbc}} \mathbb{F M} \vec{\eta}_{x}\right) \\
& =\exp \left(-\frac{1}{2 T} \vec{\eta}_{x} \mathbb{K}_{\eta_{x}}^{\mathrm{pbc}} \vec{\eta}_{x}\right)
\end{aligned}
$$

where

$$
\mathbb{K}_{\eta_{x}}^{\mathrm{pbc}}=\left(\begin{array}{ccccc}
0 & \cdots & \cdots & \cdots & 0 \\
\vdots & 2 & 1 & \cdots & 1 \\
\vdots & 1 & \ddots & \ddots & \vdots \\
\vdots & \vdots & \ddots & \ddots & 1 \\
0 & 1 & \cdots & 1 & 2
\end{array}\right)
$$

describes the correlations of the increments of our interface with p.b.c. (see Fig. 3(a)). We thus observe that the increments present long-range correlations as a result of the boundary conditions. Note that if we take the approximation of the Laplacian for small values of $q$ in Eq. (9), i.e. $1-\cos q \approx \frac{1}{2} q^{2}$, we also obtain a matrix $\mathbb{K}_{\eta_{x}}^{\tilde{\mathrm{pbc}}}$ presenting long-range correlations for the increments, as shown in Fig. 3(b). 


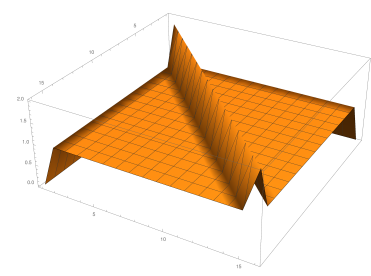

(a)

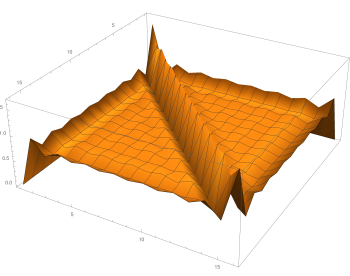

(b)
FIG. 3: (a) Correlator of the noise $\mathbb{K}_{\eta_{x}}^{\mathrm{pbc}}$ of an interface with periodic boundary conditions. (b) Correlator of the noise $\mathbb{K}_{\eta_{x}}^{\text {pbc }}$ of an interface with periodic boundary conditions with an approximation of the Laplacian as $(1-\cos q) \approx \frac{1}{2} q^{2}$ (see Eq. (9)). The increment correlations out of the diagonal persist even if we cut some Fourier modes.

\section{Spatial correlation of intervals}

To see how the intervals generated by the zeros are correlated we compute the correlation function

$$
C(r)=\left\langle\ell_{i} \ell_{i+r}\right\rangle-\left\langle\ell_{i}\right\rangle\left\langle\ell_{i+r}\right\rangle
$$

where the average is made over all the $N$ intervals of the interface.

For the correlation we observe that for odd values of $r$, the intervals are weakly anti-correlated for small values of $r$ but converge to zero almost immediately. However, for even values of $r$ the intervals are strongly anti-correlated (except for $r=0$ ) and tend more slowly to zero as observed in Fig. 4 where we plot $|C(r)|$ for $r$ even. The decay of the correlations is found to be close to an exponential behavior. Moreover the correlation function obeys a scaling law $|C(r)|=L\left|\hat{C}\left(r / L^{1 / 2}\right)\right|$ as illustrated in the inset of Fig. 4.

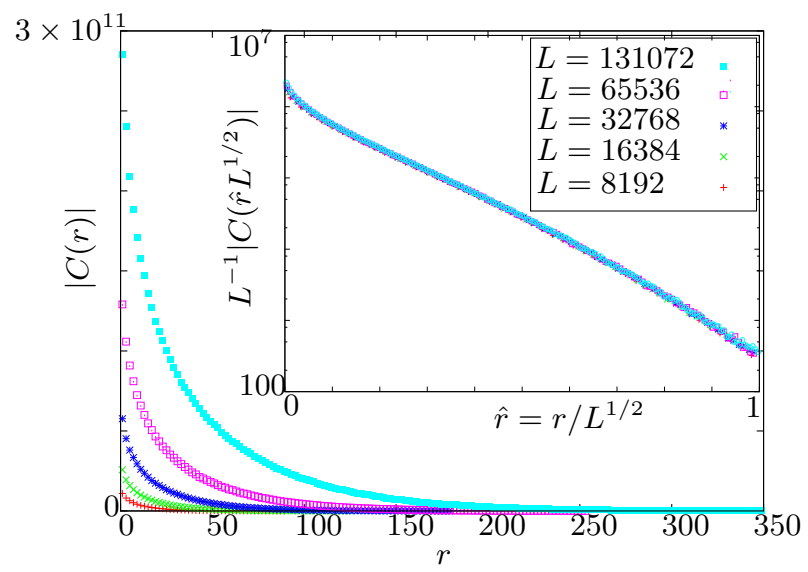

FIG. 4: Absolute value of the interval correlation $C(r)$ as defined in Eq. (11) for different sizes $L$ of the system and $r$ even. $|C(r)|$ seems to follow an exponential law $\sim e^{-r / L^{1 / 2}}$. Here we show the rescaled correlation which follows a scaling law $|C(r)|=L\left|\hat{C}\left(r / L^{1 / 2}\right)\right|$, this rescaled law being independent of $L$ (inset figure).

\section{NON-STATIONARY STATE}

The simplest equation to describe the evolution in time of a rough interface is the well-known Edwards-Wilkinson (EW) equation defined in continuous space and time in Eq. (1). In this section we will focus on a general discretized version of this equation and obtain a stability criterion that generalizes the well-known Von Neumann stability $^{30}$. This criterion establishes the necessary condition for the solution to be stable given the discretization scheme chosen.

We denote $u_{q}(t)$ as the Fourier transform of the height of the interface $u_{x}(t)$, here again we write explicitly the time dependence. For the discretization of the EW equation, the time derivative can be discretized by taking a proportion $a u_{q}(t)$ of the function at the current step and $(1-a) u_{q}(t+\Delta t)$ of the function one step later, with $a \in[0,1]$. This results in a general form of the discretized EW equation as follows

$$
u_{q}(t+\Delta t)=\frac{\left[1-a K_{q}\right] u_{q}(t)+\sqrt{\tilde{T} \Delta t} \eta_{q}(t)}{1+(1-a) K_{q}}
$$

where $\tilde{T}=T L$ and $K_{q}=c \Delta t(2(1-\cos q))^{\alpha}$ the Laplacian with roughness coefficient $\zeta=\alpha-\frac{1}{2} . K_{q}$ can be approximated as $c \Delta t q^{2 \alpha}$ for small values of $q$.

In this equation we recognize the Itō and Stratonovich discretization when choosing $a=1$ and $a=1 / 2$, respectively. As we will discuss below, the choice of the time-discretization parameter $a$ is rather important: it influences the form of steady-state itself, and the stability of the numerical scheme.

\section{A. Discrete-time solution}

The solution $u_{q}(t)$ of the continuous EW equation in Fourier space (See Eq. (1)) given by the expression (2) is obtained by direct integration of Eq. (1). By introducing $v_{q}(t)=f_{q}(t) u_{q}(t)$ such solution is simply the integral of $\partial_{t}\left(v_{q}(t)\right)=\partial_{t}\left(f_{q}(t) u_{q}(t)\right)=e^{c q^{2} t} \eta_{q}(t)$. Notice that by considering a step $\Delta t$ we have that $v(t+\Delta t)-v(t) \approx$ $\Delta t e^{c q^{2} t} \eta_{q}(t)=g(t)$ with $g(t)$ a function that does not depend explicitly on $u_{q}(t)$. In the following we find a similar solution for the discrete equation (12) written as a geometric sum as in Equation (14) below. We provide the details of the computations since it allows to pinpoint the precise origin of the stability from the convergence of a geometric sum.

Equation (12) corresponds to the most general discretization of the EW equation in Fourier space for which the discretization is controlled by the parameter $a$. Let us rewrite Eq. (12) as follows

$$
u_{q}(t+\Delta t)=A_{q} u_{q}(t)+B_{q}(t),
$$


with $A_{q}$ and $B_{q}(t)$ given by

$$
A_{q}=\frac{1-a K_{q}}{1+(1-a) K_{q}}
$$

and

$$
B_{q}(t)=\frac{\sqrt{\tilde{T} \Delta t} \eta_{q}(t)}{1+(1-a) K_{q}}
$$

where $a \in[0,1]$ and $K_{q}=c \Delta t(2(1-\cos q))^{\alpha}$ or for small values of $q, K_{q}=c \Delta t q^{2 \alpha}$ with $\alpha=\frac{1}{2}+\zeta$ for any roughness exponent $\zeta$.

We would like to find $f_{q}(t)$ so that we can express $v_{q}(t)=f_{q}(t) u_{q}(t)$ as for the continuous case and from here to find $u_{q}(t)$ that satisfies Eq. (13).

To start with, we seek a function $g(t)$ independent of $u_{q}(t)$ such that $v_{q}(t+\Delta t)-v_{q}(t)=g(t)$. This holds if $A_{q} f(t+\Delta t)-f(t)=0$, i.e. $f(t)=$ $A_{0} A_{q}^{-t / \Delta t}$. Notice that $f(t)$ can also be written as $f(t)=A_{0} e^{-\frac{t}{\Delta t} \log \frac{1-a K_{q}}{1+(1-a) K_{q}}} \approx A_{0} e^{t c q^{2}+\mathcal{O}(\Delta t)}$ (we used the approximation for small values of $q$ and $\zeta=1 / 2$ ). Thus, in this case we recover the function $f(t)$ found in the continuous solution of the EW equation by taking the limit $\Delta t \rightarrow 0$.

For finite $\Delta t$, one has

$$
v_{q}(t+\Delta t)-v_{q}(t)=A_{q}^{-t / \Delta t} \eta_{q}(t)
$$

with $A_{0}=\frac{1+(1-a) K_{q}}{\sqrt{\tilde{T} \Delta t}} A_{q}$. Then we can find the solution of $v(t)$ as follows

$$
v_{q}(t)=\sum_{t^{\prime}=0}^{t-\Delta t} A^{-t^{\prime} / \Delta t} \eta_{q}\left(t^{\prime}\right)+A_{0} v_{q}(0)
$$

with the step in the sum of size $\Delta t$. The solution $u_{q}(t)=$ $v_{q}(t) / f_{q}(t)$ in discrete time is

$$
u_{q}(t)=\frac{1}{A_{0}} \sum_{t^{\prime}=0}^{t-\Delta t} A_{q}^{\left(t-t^{\prime}\right) / \Delta t} \eta_{q}\left(t^{\prime}\right)+A_{q}^{t^{\prime} / \Delta t} u_{q}(0) .
$$

where we take $u_{q}(0)=0$ for simplicity. This is the discrete equivalent of the continuous solution (2).

\section{B. Structure factor from discrete-time solution}

We found above the discrete solution of Eq. (12). From here the structure factor can be found straightforwardly as follows

$$
S_{q}(t)=\left\langle u_{q}^{*}(t) u_{q}(t)\right\rangle=\frac{2}{A_{0}^{2}} \sum_{t^{\prime}=\Delta t}^{t} A_{q}^{2 t^{\prime} / \Delta t}
$$

where we used the fact that $\left\langle\eta_{q}(t) \eta_{q}\left(t^{\prime}\right)\right\rangle=2 \delta_{t t^{\prime}}$.
Hence,

$$
S_{q}(t)=\frac{2}{A_{0}^{2}} \frac{A_{q}^{2}\left(1-A_{q}^{2 t / \Delta t}\right)}{1-A_{q}^{2}} .
$$

Or by substituting $A_{q}$ and $A_{0}$ in the previous expression we can have the structure factor $S_{q}(t)$ in terms of $a$

$$
S_{q}(t)=\frac{2 \tilde{T} \Delta t}{K_{q}\left(2+(1-2 a) K_{q}\right)} \times F_{q}(t)
$$

with

$$
F_{q}(t)=1-\left(\frac{1-a K_{q}}{1+(1-a) K_{q}}\right)^{2 t / \Delta t}
$$

where $K_{q}$ encodes the time step $\Delta t$ and the Laplacian either with the exact expression $K_{q}=c \Delta t(2(1-\cos q))^{\alpha}$ or the approximation for small values of $q: K_{q}=c \Delta t q^{2 \alpha}$ with $\alpha=\frac{1}{2}+\zeta$ for any roughness exponent $\zeta$.

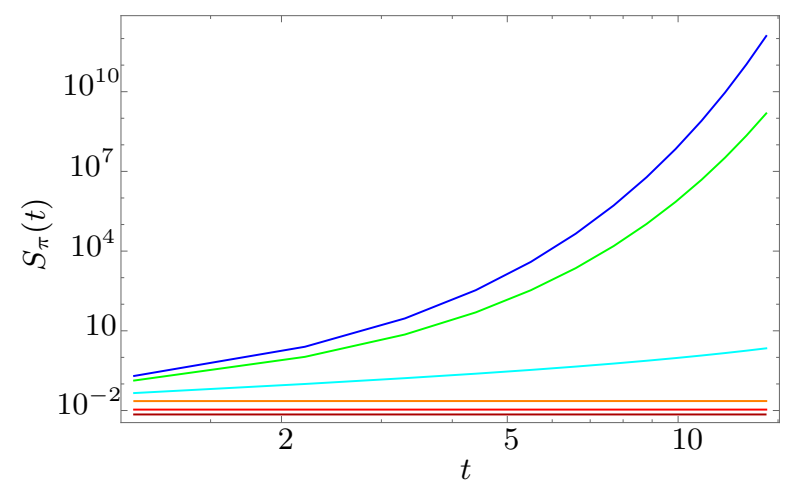

FIG. 5: Stability of $S_{q}(t)$ as a function of time with $q=q^{*}=$ $\pi, \Delta t=1.1>\Delta t_{c}$ and parameters $c=1, \zeta=1 / 2 . \quad S_{\pi}(t)$ is stable for all times for $a \leq 1 / 2(a=0,0.25,0.5$ in dark red, red and orange, respectively, from bottom up), however if $a$ becomes larger ( $a=1,0.95,0.75$ in blue, green and cyan, respectively, from top down) $S_{\pi}(t)$ loses stability

The convergence of Eq. (16) in the limit $t \rightarrow \infty$ is guaranteed since $F_{q}(t)$ given by (17) converges to 1 for any value of $a$ and $q$. In this limit the structure factor $S_{q}=S_{q}(t \rightarrow \infty)$ takes the following form

$$
S_{q}=\frac{\tilde{T} \Delta t}{K_{q}\left(1+\frac{(1-2 a)}{2} K_{q}\right)}
$$

with some particular cases corresponding to 'anti-Itō', Stratonovich and Itō discretizations for $a=0,1 / 2$ and 1 , respectively, which yield

$$
\begin{aligned}
S_{q} & =\frac{\tilde{T} \Delta t}{K_{q}\left(1+\frac{1}{2} K_{q}\right)} & & a=0 \\
S_{q} & =\frac{\tilde{T} \Delta t}{K_{q}} & a & =1 / 2 \\
S_{q} & =\frac{\tilde{T} \Delta t}{K_{q}\left(1-\frac{1}{2} K_{q}\right)} & a & =1
\end{aligned}
$$


where $K_{q}=c \Delta t(2(1-\cos q))^{\alpha}$ or $K_{q}=c \Delta t q^{2 \alpha}$ with $\alpha=\frac{1}{2}+\zeta$ for any roughness exponent $\zeta$. In particular, the choice $a=1 / 2$ given by expression (20) cancels out the correction for $\Delta t$ in the structure factor. This constitutes one of our main results: the Stratonovitch discretization $(a=1 / 2)$ is the discretization which minimizes the influence of the time step $\Delta t$ on the steady state.

Notice that in Eq. (18), a wrong choice of $\Delta t$ can make the denominator equal to zero, this happens whenever $1+\frac{(1-2 a)}{2} K_{q}=0$, i.e. if

$$
\Delta t_{c}=\frac{2}{c(2 a-1)(2(1-\cos q))^{\alpha}}
$$

for $a>1 / 2$ and $K_{q}=c \Delta t(2(1-\cos q))^{\alpha}$. For the explicit solution associated to the parameter $a=1$ (Itō discretization) it is known from general experience that the solution of the EW equation is unstable if the time step is too large: this is indeed the Von Neumann stability criterion ${ }^{30}$. Our analysis hence provides a detailed understanding of this stability for any $a$ : the relation (22) gives the critical value of $\Delta t$ for which $S_{q}$ becomes apparently negative as a result of the divergence of the geometric series (15). The expression (22) thus represents the threshold above which the numerical procedure becomes unstable.

The smallest value that $\Delta t_{c}$ can take corresponds to the mode associated to $q^{*}=\pi$ for which

$$
\Delta t_{c}\left(q^{*}\right)=\frac{2}{(2 a-1) 4^{\alpha} c}
$$

for $a>1 / 2$ and $\alpha=\frac{1}{2}+\zeta$ for any roughness exponent $\zeta$. This implies that the modes related to shorter distances are the first to become unstable. Fig. 5 shows the stability of $S_{q}(t)$ for different values of $a$ and a choice of $\Delta t=2.1>\Delta t_{c}$ with $q *=\pi$ and $c=1, \zeta=1 / 2$. The most important consequence of our analysis is that the structure factor $S_{\pi}(t)$ is stable at all times for any value $a \leq 1 / 2$ of the discretization parameter, while for larger values of $a$ it can lose stability for large enough time step $\Delta t$ given by expression (23).

\section{Structure factor from trajectorial probabilities}

By computing the stationary distribution $P^{\mathrm{st}}\left[u_{q}(t)\right]$ and comparing it with the probability of the reversed process we can obtain the structure factor $S_{q}$ in the stationary state. The dynamics of the process is not reversible since the normal process $u_{q}(t)$ and the same process reversed in time are described with Itō $(a=1)$ and anti-Itō $(a=0)$ discretization, respectively. We compare the probability of the process with the distribution of trajectories $P^{\text {traj }}\left[u_{q}(t), a\right]$ and the probability of the trajectories reversed in time $P^{\text {traj, } \mathrm{R}}\left[u_{q}(t), 1-a\right]$, whose temporal symmetry is conserved by the transformation $a \mapsto 1-a$. The probabilities of the process and of the trajectories compare as follows

$$
\frac{P^{\mathrm{st}}\left[u_{q}\left(t_{f}\right)\right]}{P^{\mathrm{st}}\left[u_{q}\left(t_{0}\right)\right]}=\frac{P^{\mathrm{traj}}\left[u_{q}(t), a\right]}{P^{\mathrm{traj}, \mathrm{R}}\left[u_{q}(t), 1-a\right]} .
$$

The probability of a trajectory of $u$ will be deduced from that of the noise as

$$
P^{\text {traj }}\left[u_{q}(t)\right] \propto \exp \left(-\frac{1}{2} \frac{1}{2 \tilde{T} \Delta t} \sum_{t=0}^{t_{f}} \sum_{q} \eta_{q}(t) \eta_{-q}(t)\right) .
$$

In this case we focus on the implicit form of the equation (12) with $a=0$, which it is widely used when working with numerical simulations due to its stability. This equation takes the following form

$$
u_{q}(t)=\frac{u_{q}(t+\Delta t)+\sqrt{\tilde{T} \Delta t} \eta_{q}(t)}{1+\Delta t c q^{2}},
$$

where we use the approximation of the Laplacian for small $q$.

From Eqs. (25) and (26) we can express $\eta_{q}(t)$ and $\eta_{q}^{\mathrm{R}}(t)$ in terms of $u_{q}(t+\Delta t)$ and $u_{q}(t)$ to compute the right hand side of Eq. (24). For the forward trajectory we use $-\eta_{q}(t)=\left[u_{q}(t+\Delta t)-u_{q}(t)+\Delta t c q^{2} u_{q}(t)\right] / \sqrt{\tilde{T} \Delta t}$ and for the trajectory reversed in time we look at $-\eta_{q}^{\mathrm{R}}(t)=$ $\left[u_{q}(t+\Delta t)-u_{q}(t)-\Delta t c q^{2} u_{q}(t)\right] / \sqrt{\tilde{T} \Delta t}$ corresponding to $a=1$ in Eq. (12). The factor in the sum on the right hand side in Eq. (24) is found to be

$$
\begin{aligned}
{\left[\eta_{q}(t) \eta_{-q}(t)-\eta_{q}^{\mathrm{R}}(t) \eta_{-q}^{\mathrm{R}}(t)\right] } & =2 \Delta t c q^{2}\left(1+\frac{1}{2} \Delta t c q^{2}\right) \\
\times u_{q}(t+\Delta t) u_{-q}( & t+\Delta t)-u_{q}(t) u_{-q}(t) \\
& =2 \Delta t c\left\langle u_{q}^{*}(t) u_{q}(t)\right\rangle \\
& \times\left[u_{q}\left(t^{\prime}\right) u_{-q}\left(t^{\prime}\right)\right]_{t}^{t+\Delta t}
\end{aligned}
$$

where we denote $\left[u_{q}\left(t^{\prime}\right) u_{-q}\left(t^{\prime}\right)\right]_{t}^{t+\Delta t}=u_{q}(t+\Delta t) u_{-q}(t+$ $\Delta t)-u_{q}(t) u_{-q}(t)$. From the last expression (27) we can identify the structure factor $S_{q}=S_{q}(t \rightarrow \infty)=$ $\left\langle u_{q}^{*}(t) u_{q}(t)\right\rangle$. Therefore Eq. (24) takes the form

$\frac{P^{\mathrm{st}}\left[u_{q}\left(t_{f}\right)\right]}{P^{\mathrm{st}}\left[u_{q}\left(t_{0}\right)\right]}=\exp \left(-\frac{1}{2} \sum_{t=0}^{t_{f}-\Delta t} \sum_{q} S_{q}^{-1}\left[u_{q}\left(t^{\prime}\right) u_{-q}\left(t^{\prime}\right)\right]_{t}^{t+\Delta t}\right)$

with the structure factor in the stationary state given by

$$
S_{q}=\frac{\tilde{T}}{c q^{2}\left(1+\frac{1}{2} c \Delta t q^{2}\right)} .
$$

Note that this expression for the structure factor differs from the common expression $S_{q}=1 / q^{2}$ since it contains a correction term proportional to the time step $\Delta t$ induced by the time discretization. Equation (28) is in agreement with expression (19) found in the previous section with the choice $a=0$ and $K_{q}=c \Delta t q^{2}$. 


\section{Structure factor. Numerical simulations}

For the evolution of the structure factor $S_{q}(t)$ (see Fig. 6) we find a characteristic $q_{c}$, such that for $q<q_{c}$ $S_{q}(t)$ saturates to a plateau whose value depends on $t$, while the large $q$ behavior is independent of $t$. This can also be compared with the analytic expression (16).

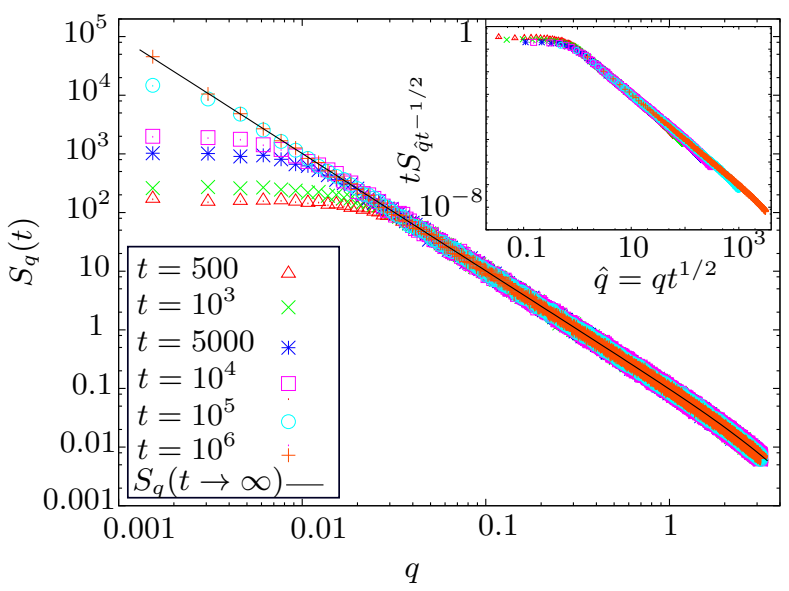

FIG. 6: Evolution of structure factor in time with discretization parameter $a=0$ in the evolution equation (12) with roughness exponent $\zeta=1 / 2$. The bending tail of $S_{q}(t)$ for $q$ close to $\pi$ is completely understood by means of the correction term proportional to $\Delta t$ and the approximate Laplacian encoded in the variable $K_{q}=c \Delta t q^{2}$ as derived in Eq. (19) (solid line). A dynamic scaling law is found to be $S_{q}=t^{-1} \hat{S}_{q t^{1 / 2}}$ (inset).

If we scale the structure factor as $S_{q}=t^{-1} \hat{S}_{q t^{1 / 2}}$ all the curves collapse in a single curve (see Fig. 6). This is in perfect agreement with the analytic expression found in Eq. (18). It also shows that the non-steady relaxation of the interface is governed by a dynamical length growing as $L_{\mathrm{dyn}}(t) \sim t^{1 / 2}$. In other words, we can write $S_{q} \equiv q^{2} \tilde{S}_{q L_{\mathrm{dyn}}(t)}$, such that large lengthscales $q<2 \pi / L_{\text {dyn }}(t)$ are out of equilibrium and retain memory of the flat initial condition $S_{q} \sim\left(2 \pi / L_{\mathrm{dyn}}(t)\right)^{-2}$, while small lengthscales $q>2 \pi / L_{\mathrm{dyn}}(t)$ are equilibrated and display the characteristic equilibrium roughness exponent $S_{q} \sim q^{-2}$. Equilibration is thus expected for times $t \gtrsim t_{\mathrm{sat}} \sim L^{2}$, as $L_{\mathrm{dyn}}(t) \rightarrow L$.

The tail of the structure factor $S_{q}(t)$ for $q$ close to $\pi$ (See Fig. 6) is controlled by the correction term proportional to $\Delta t$, and is strongly influenced by the choice of the parameter $a$ in the evolution equation (12) and of the Laplacian encoded in the variable $K_{q}$ in Eq. (18). In the simulations we used $a=0$ and $\tilde{T}=0.1$ and the approximate Laplacian $K_{q}=c \Delta t q^{2}$ with $c=1$ and $\Delta t=0.1$.

\section{E. Density of zeros}

Another observable is the non-stationary density $\rho$ of crossing zeros. The initial condition in the out-of- equilibrium state is a flat interface. Immediately after we let the interface evolve, we observe that a large number of zeros appear. When the interface realizes it is finite, i.e. at a saturation time $t_{\text {sat }} \sim L^{2}$, the density of zeros reaches a stationary state as shown in Fig. 7. A scaling law can be found, it scales as $\rho(L, t)=L^{-1 / 2} \hat{\rho}\left(t / L^{2}\right)$ for which a perfect collapse is found as shown in Fig. 7.

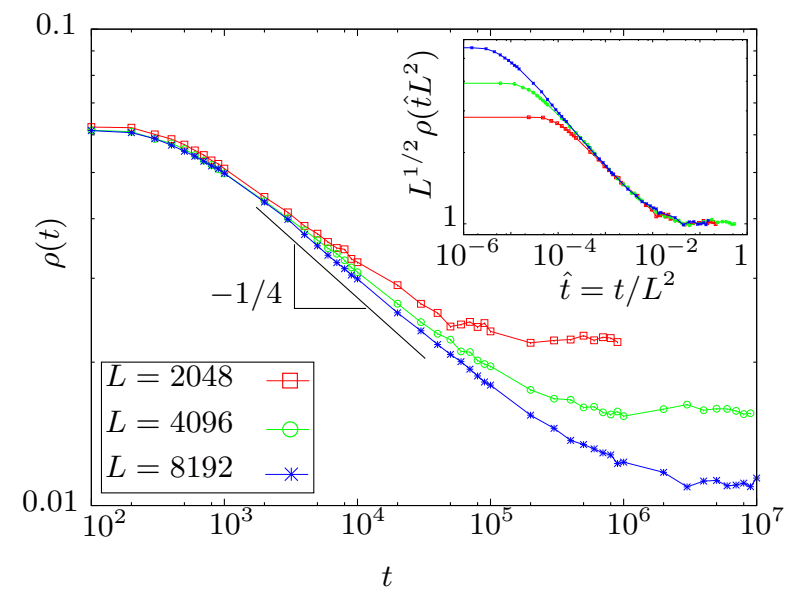

FIG. 7: Evolution of density of zeros in time for different system sizes: $L=2048,4096,8192$ (in red, green and blue, respectively), averaged over 1000 realizations. There is a characteristic time $t_{c} \sim L^{2}$ from which the density reaches a steady state. The scaling law for the density of zeros is found to be $\rho(L, t)=L^{-1 / 2} \hat{\rho}\left(t / L^{2}\right)$.

We can extract a power law from the behavior of the density of zeros before the saturation time $t_{\text {sat }}$ which turns out to be $\rho(t) \sim t^{-1 / 4}$. This exponent is validated from the scaling found before since $\rho(L, t)=$ $L^{-1 / 2}\left(t / L^{2}\right)^{-1 / 4}=t^{-1 / 4}$. We can also see that the regime after the saturation time behaves as $\rho(L) \sim$ $L^{-1 / 2}$. This is consistent with the finite-size scaling for $p(\ell)$ shown in Fig. 2. Since $\langle\ell\rangle \equiv \sum_{\ell=1}^{L} p(\ell) \ell \approx$ $\int_{0}^{L} \ell^{-3 / 2} \ell d \ell \sim L^{1 / 2}$, we get that $\rho(L)=\langle\ell\rangle^{-1} \sim L^{-1 / 2}$. The density of zeros thus vanishes in the thermodynamic limit due to the infrared divergence of $\langle\ell\rangle$. This is directly related to the power-law decay exponent of $p(\ell)$ here $\gamma=3 / 2$, as predicted by the Sparre-Andersen theorem.

As discussed in relation with $S_{q}(t)$, the scaling law for $\rho(L, t)$ is consistent with a relaxation dominated by a single dynamical length $L_{\text {dyn }}(t) \sim t^{1 / 2}$. We can thus write $\rho(L, t)=L^{-1 / 2} \tilde{\rho}\left(L_{\mathrm{dyn}}(t) / L\right)$, such that $\tilde{\rho}(x) \sim x^{1 / 2}$ for $x \ll 1$, corresponding to the non-steady regime, and $\tilde{\rho}(x) \sim$ const. for large $x \gg 1$, corresponding to the equilibrated regime. Comparing $\rho(t) \sim L_{\text {dyn }}(t)^{-1 / 2} \sim t^{-1 / 4}$, valid for intermediate times, with $\rho(t \rightarrow \infty) \sim L^{-1 / 2}$ we can see that finite-size scaling in the steady-state directly translates into the non-stationary finite-time scaling, by replacing $L \rightarrow L_{\mathrm{dyn}}(t)$. In particular the power-law decay exponent $1 / 4$ in the density of zeros is related to the dynamical exponent $z=2$ in $L_{\mathrm{dyn}}(t) \sim t^{1 / z}$ and the Sparre-Andersen exponent $\gamma=3 / 2$ as $(2-\gamma) / z=1 / 4$. 
Interestingly, this let us predict that the non-stationary distribution of intervals can be expressed as $p(\ell, t) \sim$ $\ell^{-3 / 2} \tilde{p}\left(\ell t^{-1 / 2}\right)$ for $t<t_{\text {sat }}$.

\section{CONCLUSIONS}

In this paper we have focused on fluctuating interfaces that belong to the EW universality class, i.e. interfaces with roughness coefficient $\zeta=1 / 2$. We investigated the spatial first-passage probability which was obtained from the length of the intervals generated by the crossing zeros of the interface with respect to its average. The linearity of the Langevin equation ensures that the symmetry $u_{x}(t) \rightarrow-u_{x}(t)$ is conserved, therefore the persistence exponent for the positive and negative intervals is exactly the same ${ }^{15}$, i.e. $\theta_{s}^{+}=\theta_{s}^{-}$. This justifies the fact that for stationary interfaces the distribution of intervals obtained numerically was obtained without making a distinction between the positive and the negative intervals. Regarding the distribution of intervals, both positive and negative, we have shown the agreement of the SparreAndersen theorem for random walks which measures the persistence in time with the spatial distribution of intervals for which the height in an interval is positively or negatively persistent. We also found a scaling function for such distribution related with the finite system size $L$ for which intervals larger than a certain $\ell_{\max } \sim L / 2$ are rare. We investigated the correlations between the intervals from which we obtained a scaling function with the system size $L$. The influence of periodic boundary conditions was also studied by means of the increments correlation of the interface itself.

Concerning the non-stationary regime we have presented a general discretization for the linear Langevin equation that simulates the evolution of fluctuating interfaces with roughness coefficient $\zeta$. An exact expression for the evolution of the structure factor was obtained and, surprisingly, it depends on the time step $\Delta t$ and on the discretization parameter $a$ even in the infinitetime limit. The correction in $\Delta t$ that it implies for the natural expression of the structure factor disappears for Stratonovich discretization corresponding to the choice of our parameter $a=1 / 2$. We have also found a relation that establishes the critical value of the time step $\Delta t_{c}$ needed as a function of the parameter $a$ so that stability of the simulation is guaranteed. Finally we study numerically the evolution of the structure factor $S_{q}(t)$ and the density $\rho$ of zeros . Regarding $S_{q}(t)$ two regimes were found, before a critical value $q_{c} \sim t^{1 / 2}$ we observe a plateau for small $q$ and for larger values of $q>q_{c} S_{q}(t)$ presents a power-law decay which goes as $\sim q^{2}$. The stationary limit found numerically is in perfect agreement with the analytic expression found for $S_{q}(t \rightarrow \infty)$. For the density of zeros $\rho$ two regimes were found as well. Before a saturation time that scales as $t_{\text {sat }} \sim L^{2}$ the density of zeros follows a power-law with a decay that goes as $\sim t^{-1 / 4}$, which is in perfect agreement with the scaling of reaction diffusion processes found in the literature ${ }^{34}$, and for times larger than $t_{\text {sat }}$ the density reaches a stationary state as expected.

\section{Acknowledgements}

The authors acknowledge the ECOS project A12E05 which allowed this collaboration. ABK wishes to thank the European Union EMMCSS visiting scientist program and acknowledges partial support from Projects PIP11220090100051 and PIP11220120100250CO (CONICET). VL acknowledges the Coopinter project EDC25533 for partial funding. ALZ wishes to thank the hospitality of the Condensed Matter Theory Group at Centro Atómico Bariloche, S.C. de Bariloche, Argentina, and acknowledges funding from the Mexican National Council for Science and Technology (CONACyT).
1 S. Redner, A Guide to First-Passage Processes (Cambridge University Press, 2001), ISBN 978-0-521-65248-3.

2 E. W. Montroll and G. H. Weiss, Journal of Mathematical Physics 6, 167 (1965), ISSN 0022-2488, 10897658, URL http://scitation.aip.org/content/aip/ journal/jmp/6/2/10.1063/1.1704269.

3 H. P. J. McKean, J. Math. Kyoto Univ. 2, 227 (1962), ISSN 0023-608X, URL http://projecteuclid. org/euclid.kjm/1250524936.

4 Y. G. Sinai, Theor Math Phys 90, 219 (1992), ISSN 0040-5779, 1573-9333, URL http://link.springer.com/ article/10.1007/BF01036528.

5 Y. G. Sinai, Commun.Math. Phys. 148, 601 (1992), ISSN 0010-3616, 1432-0916, URL http://link.springer.com/ article/10.1007/BF02096550.

6 B. Derrida, A. J. Bray, and C. Godreche, J. Phys. A: Math. Gen. 27, L357 (1994), ISSN 0305-4470, URL http: //stacks.iop.org/0305-4470/27/i=11/a=002.

7 A. J. Bray, Advances in Physics 43, 357 (1994), ISSN 0001-8732, URL http://dx.doi.org/10.1080/ 00018739400101505.

${ }^{8}$ H. J. Hilhorst, Physica A: Statistical Mechanics and its Applications 277, 124 (2000), ISSN 03784371, URL http://www.sciencedirect.com/science/ article/pii/S0378437199005099.

9 D. B. Dougherty, I. Lyubinetsky, E. D. Williams, M. Constantin, C. Dasgupta, and S. Sarma, Phys. Rev. Lett. 89, 136102 (2002), URL http://link.aps.org/doi/10.1103/ PhysRevLett.89.136102.

10 J. Krug, Advances in Physics 46, 139 (1997), ISSN 0001-8732, URL http://dx.doi.org/10.1080/ 00018739700101498.

11 H. Kallabis and J. Krug, Europhysics Letters (EPL) 45, 20 (1999), ISSN 0295-5075, 1286-4854, URL 
http://stacks. iop.org/0295-5075/45/i=1/a=020? key=crossref . 14d78e6d02dec0e02f71c54cfddbc3a9.

12 C. Godrèche, Solids Far from Equilibrium (Cambridge University Press, 1991), ISBN 978-0-521-41170-7.

13 A. J. Bray, S. N. Majumdar, and G. Schehr, Advances in Physics 62, 225 (2013), ISSN 0001-8732, URL http: //dx.doi.org/10.1080/00018732.2013.803819.

14 S. N. Majumdar and A. J. Bray, Phys. Rev. Lett. 86, 3700 (2001), URL http://link.aps.org/doi/10.1103/ PhysRevLett.86.3700.

15 M. Constantin, S. Das Sarma, and C. Dasgupta, Phys. Rev. E 69, 051603 (2004), URL http://link.aps.org/ doi/10.1103/PhysRevE.69.051603.

16 C. Sire, Phys. Rev. E 78, 011121 (2008), URL http:// link.aps.org/doi/10.1103/PhysRevE.78.011121.

17 P. A. Alemany and D. ben Avraham, Physics Letters A 206, 18 (1995), ISSN 0375-9601, URL http://www.sciencedirect.com/science/article/ pii/037596019500625D.

18 B. Derrida, V. Hakim, and R. Zeitak, Phys. Rev. Lett. 77, 2871 (1996), URL http://link.aps.org/doi/10.1103/ PhysRevLett.77.2871.

19 S. N. Majumdar, C. Sire, A. J. Bray, and S. J. Cornell, Phys. Rev. Lett. 77, 2867 (1996), URL http://link.aps . org/doi/10.1103/PhysRevLett.77.2867.

20 M. Constantin, C. Dasgupta, P. P. Chatraphorn, S. N. Majumdar, and S. Das Sarma, Phys. Rev. E 69, 061608 (2004), URL http://link. aps .org/doi/10.1103/ PhysRevE.69.061608.

21 J. McFadden, IRE Transactions on Information Theory $\mathbf{2}$, 146 (1956), ISSN 0096-1000.

22 J. McFadden, IRE Transactions on Information Theory 4, 14 (1958), ISSN 0096-1000.
23 J. Lamperti, Transactions of the American Mathematical Society 104, 62 (1962), ISSN 0002-9947, URL http:// www.jstor.org/stable/1993933.

24 S. N. Majumdar and C. Sire, Phys. Rev. Lett. 77, 1420 (1996), URL http://link.aps.org/doi/10.1103/ PhysRevLett.77.1420.

25 K. Oerding, S. J. Cornell, and A. J. Bray, Phys. Rev. E 56, R25 (1997), URL http://link.aps.org/doi/10. 1103/PhysRevE.56.R25.

26 J. Krug, H. Kallabis, S. N. Majumdar, S. J. Cornell, A. J. Bray, and C. Sire, Phys. Rev. E 56, 2702 (1997), URL http://link.aps.org/doi/10.1103/PhysRevE.56.2702.

27 A. L. Zamorategui, V. Lecomte, and A. B. Kolton, In preparation (2016).

28 D. Chakraborty and J. K. Bhattacharjee, Phys. Rev. E 75, 011111 (2007), URL http://link.aps.org/doi/10.1103/ PhysRevE.75.011111.

29 E. S. Andersen, Math. Scand 1, 1954 (1953).

${ }^{30}$ W. H. Press, Numerical Recipes 3rd Edition: The Art of Scientific Computing (Cambridge University Press, 2007), ISBN 978-0-521-88068-8.

31 K. Ito, Proceedings of the Imperial Academy 20, 519 (1944).

32 R. L. Stratonovich, Topics In the Theory of Random Noise (CRC Press, 1967), ISBN 978-0-677-00790-8.

33 W. Krauth, Statistical Mechanics: Algorithms and Computations (Oxford University Press, UK, 2006), ISBN 9780-19-851535-7.

34 D. Toussaint and F. Wilczek, The Journal of Chemical Physics 78, 2642 (1983), ISSN 0021-9606, 10897690, URL http://scitation.aip.org/content/aip/ journal/jcp/78/5/10.1063/1.445022. 